\title{
GENERATORS OF $\mathrm{II}_{1}$ FACTORS
}

\author{
Ken Dykema, Allan Sinclair, Roger Smith and Stuart White
}

Abstract. In 2005, Junhao Shen introduced a new invariant, $\mathscr{G}(N)$, of a diffuse von Neumann algebra $N$ with a fixed faithful trace, and he used this invariant to give a unified approach to showing that large classes of $\mathrm{II}_{1}$ factors $M$ are singly generated. This paper focuses on properties of this invariant. We relate $\mathscr{G}(M)$ to the number of self-adjoint generators of a $\mathrm{II}_{1}$ factor $M$ : if $\mathscr{G}(M)<n / 2$, then $M$ is generated by $n+1$ self-adjoint operators, whereas if $M$ is generated by $n+1$ self-adjoint operators, then $\mathscr{G}(M) \leqslant n / 2$. The invariant $\mathscr{G}(\cdot)$ is well-behaved under amplification, satisfying $\mathscr{G}\left(M_{t}\right)=t^{-2} \mathscr{G}(M)$ for all $t>0$. In particular, if $\mathscr{G}\left(\mathscr{L} \mathbb{F}_{r}\right)>0$ for any particular $r>1$, then the free group factors are pairwise non-isomorphic and are not singly generated for sufficiently large values of $r$. Estimates are given for forming free products and passing to finite index subfactors and the basic construction. We also examine a version of the invariant $\mathscr{G}_{\mathrm{sa}}(M)$ defined only using self-adjoint operators; this is proved to satisfy $\mathscr{G}_{\mathrm{sa}}(M)=2 \mathscr{G}(M)$. Finally we give inequalities relating a quantity involved in the calculation of $\mathscr{G}(M)$ to the free-entropy dimension $\delta_{0}$ of a collection of generators for $M$.

Mathematics subject classification (2000): 46L10, 46L54.

Keywords and phrases: $\mathrm{II}_{1}$ factors, generators, generator invariant, free orbit dimension.

\section{REFERENCES}

[1] R. G. Douglas And C. Pearcy, Von Neumann algebras with a single generator, Michigan Math. J., 16:21-26, 1969.

[2] K. DyKema AND U. HAaGerup, Invariant subspaces of the quasinilpotent DT-operator, J. Funct. Anal., 209(2):332-366, 2004.

[3] K. J. DYKeMA, Free products of hyperfinite von neumann algebras and free dimension, Duke Math. J., 69(1):97-119, 1993.

[4] K. J. DYKEMA, Interpolated free group factors, Pacific J. Math., 163(1):123-135, 1994.

[5] K. J. DYKEMA AND U. HAAGERUP, DT-operators and decomposability of Voiculescu's circular operator, Amer. J. Math., 126(1):121-189, 2004.

[6] K. J. Dykema, K. Jung, And D. Shlyakhtenko, The microstates free entropy dimension of any DT-operator is 2, Doc. Math., 10:247-261 (electronic), 2005.

[7] L. GE, Applications of free entropy to finite von neumann algebras, Amer. J. Math., 119(2):467-485, 1997.

[8] L. GE AND S. POPA, On some decomposition properties for factors of type $\mathrm{II}_{1}$, Duke Math. J., 94(1):79-101, 1998.

[9] L. GE AND J. SHEN, Generator problem for certain property $T$ factors, Proc. Natl. Acad. Sci. USA, 99(2):565-567 (electronic), 2002.

[10] D. HADWIN AND J. SHEN, Free orbit dimension of finite von Neumann algebras, J. Funct. Anal., 249(1):75-91, 2007.

[11] V. F. R. JonES, Index for subfactors, Invent. Math., 72(1):1-25, 1983.

[12] K. Jung, A free entropy dimension lemma, Pacific J. Math., 211(2):265-271, 2003.

[13] K. Jung, The free entropy dimension of hyperfinite von Neumann algebras, Trans. Amer. Math. Soc., 355(12):5054-5089, 2003.

[14] K. JunG, Strongly 1-bounded von neumann algebras, Geom. Func. Anal., 17 (4):1180-1200, 2007. 
[15] K. Jung, A hyperfinite inequality for free entropy dimension, Proc. Amer. Math. Soc., 134(7):2099-2108 (electronic), 2006.

[16] C. PEARCY, $W^{*}$-algebras with a single generator, Proc. Amer. Math. Soc., 13:831-832, 1962.

[17] F. RĂDULESCU, The fundamental group of the von Neumann algebra of a free group with infinitely many generators is $\mathbb{R}_{+}$, J. Amer. Math. Soc., 5(3):517-532, 1992.

[18] F. RĂDULESCU, Random matrices, amalgamated free products and subfactors of the von Neumann algebra of a free group, of noninteger index, Invent. Math., 115(2):347-389, 1994.

[19] J. SHEN, Singly generated $\mathrm{II}_{1}$ factors, J. Operator Theory, to appear. arXiv:math.OA/0511327, 2005.

[20] A. M. SinClair AND R. R. SMITH, Finite von Neumann algebras and masas, Volume 351 of London Mathematical Society Lecture Notes Series, Cambridge University Press, Cambridge, 2008.

[21] S. J. SZAREK, Nets of Grassmann manifold and orthogonal group, In Proceedings of research workshop on Banach space theory (Iowa City, Iowa, 1981), pages 169-185, Iowa City, IA, 1982. Univ. Iowa.

[22] D. M. TopPING, Lectures on von Neumann algebras, Number 36 in Van Nostrand Reinhol Mathematical Studies. Van Nostrand, London, 1971.

[23] D. VoICULESCU, The analogues of entropy and of Fisher's information measure in free probability theory, II, Invent. Math., 118(3):411-440, 1994.

[24] D. VoICUlEscu, The analogues of entropy and of Fisher's information measure in free probability theory, III, The absence of Cartan subalgebras, Geom. Funct. Anal., 6(1):172-199, 1996.

[25] D. VoICULESCU, K. J. DYKEMA, AND A. NICA, Free Random Variablees, volume 1 of CRM Monograph, American Mathematical Society, Providence, 1992.

[26] W. Wogen, On generators for von Neumann algebras, Bull. Amer. Math. Soc., 75:95-99, 1969. 